\title{
Effect of multidisciplinary team care on the management of cirrhotic patients with upper gastrointestinal bleeding: a retrospective cohort study
}

\author{
Songliu Wang ${ }^{1}$, Xiangyun Qian ${ }^{2}$ \\ ${ }^{1}$ Department of Gastroenterology, Nantong Haimen People's Hospital, Nantong, China; ${ }^{2}$ Nursing Department, Third Affiliated Hospital of \\ Nantong University, Nantong, China \\ Contributions: (I) Conception and design: S Wang, X Qian; (II) Administrative support: X Qian; (III) Provision of study materials or patients: \\ S Wang; (IV) Collection and assembly of data: S Wang; (V) Data analysis and interpretation: S Wang; (VI) Manuscript writing: All authors; (VII) \\ Final approval of manuscript: All authors. \\ Correspondence to: Xiangyun Qian. Nursing Department, Third Affiliated Hospital of Nantong University, No. 60 Qingnian Zhong road, Chongchuan \\ District, Nantong 226006, China. Email: qxy03062020@126.com.
}

Background Multidisciplinary team care (MDTC) has been proposed to improve the prognosis and
quality of life of patients through the collaboration of multiple disciplines. However, it is still unclear
whether MDTC is effective in the management of cirrhotic patients with upper gastrointestinal bleeding
(UGIB).

Methods: This was a retrospective cohort study enrolling cirrhotic patients with UGIB receiving traditional care or MDTC between July 2015 and December 2019. Clinical data and laboratory test results of enrolled patients were collected by 2 independent investigators. The primary outcomes were mortality and the incidence of rebleeding within 1 year. Furthermore, the quality of life of enrolled patients was chosen as the secondary outcome. Multivariable logistic regression was used to determine the risk factors of mortality and rebleeding, after adjusting for confounding variables by univariable logistic regression. Also, multivariable linear regression was used to determine the effects of MDTC on the quality of life of enrolled patients.

Results: This study finally included 206 cirrhotic patients with UGIB, with 101 patients in the traditional care group and 105 patients in the MDTC group. Compared with the traditional care group, patients in the MDTC group had significantly higher Child-Pugh and model for end-stage liver disease (MELD) scores ( $\mathrm{P}=0.02$ and 0.04 , respectively). Furthermore, patients in the MDTC group had a significantly lower level of white blood cells (WBC) and a significantly higher level of aspartate transaminase (AST) compared to the traditional care group $(\mathrm{P}=0.01$ and 0.02 , respectively). Multivariable logistic regression then identified MDTC as a protective factor for rebleeding and mortality within 1 year. Higher MELD scores and more required units of packed red blood cells (RBC) were associated with a higher incidence of rebleeding and mortality. Additionally, patients in the MDTC group had less discomfort and depression than those in the traditional care group (both $\mathrm{P}<0.01$ ), and MDTC was associated with improved quality of life according to the multivariable linear regression analysis.

Conclusions: MDTC, compared with traditional care, reduced the incidence of rebleeding and mortality over the long term. It was also useful for relieving anxiety and improving the quality of life of patients.

Keywords: Multidisciplinary team care (MDTC); upper gastrointestinal bleeding (UGIB); cirrhosis; mortality; rebleeding; quality of life

Submitted Dec 10, 2020. Accepted for publication Feb 05, 2021.

doi: 10.21037/apm-21-85

View this article at: http://dx.doi.org/10.21037/apm-21-85 


\section{Introduction}

Cirrhosis is caused by several factors, including hepatitis, excessive alcohol, cholestasis, and the use of certain drugs, of which hepatitis B is the main risk factor in China $(1,2)$. Cirrhosis can lead to portal hypertension, involving increased portal inflow and high portal outflow resistance (3). Portal hypertension can induce several serious complications, such as acute upper gastrointestinal bleeding (UGIB). It was reported that severe UGIB occurred in $10-30 \%$ of cirrhotic patients and had a mortality rate of $5-20 \%$ each year (4-7). The mortality of UGIB patients is high without appropriate treatment (8). Current first-line treatment for UGIB includes vasoactive drugs and endoscopic procedures to initially control bleeding and prevent early rebleeding. The survival of cirrhotic patients has significantly improved along with the developments in pharmacological treatment strategies. Despite this, the management of cirrhotic patients with UGIB still remains a clinical challenge.

It is known that the management of cirrhotic patients with UGIB is not only dependent on in-hospital care, but also the collaboration of the family. The purpose of inhospital care is to control bleeding and to take measures to prevent rebleeding, while family care can also enhance measures to prevent rebleeding. In this way, family care may be more important than in-hospital care for improving the health status and quality of life of patients (9). However, the traditional mode of care for cirrhotic patients with UGIB is only dependent on nurses, exhibiting poor accessibility and effectiveness (10).

Recently, multidisciplinary team care (MDTC), a patient-centered medical collaboration, has been proposed to provide specialized and well-rounded care for patients by a group-coordinated symposium including various experts from different disciplines. MDTC is expected to improve the prognosis and quality of life of patients by the collaboration of multiple disciplines. It has been reported to be effective in the management of several types of diseases, such as diabetes mellitus, breast cancer, and ankylosing spondylitis (10-12). However, it is still unclear whether MDTC is effective in the management of cirrhotic patients with UGIB. Therefore, the present retrospective cohort study was conducted to investigate the effects of MDTC on the management of cirrhotic patients with UGIB compared with traditional care.

We present the following article in accordance with the STROBE reporting checklist (available at http://dx.doi. org/10.21037/apm-21-85).

\section{Methods}

\section{Patient selection}

To conduct this retrospective cohort study, we recruited cirrhotic patients with UGIB from Third Affiliated Hospital of Nantong University between July 2015 and December 2019. Patients who were admitted to this hospital between July 2015 and July 2017 received traditional care and those who were admitted between August 2017 and September 2019 received MDTC. UGIB in this study was defined as hematemesis or melena. The age and sex of patients were not limited in this study. Patients were excluded if they died before receiving treatments, had hepatocellular carcinoma or other cancer, had incomplete medical records, or experienced rebleeding within 6 weeks after a previous UGIB. All procedures performed in this study involving human participants were in accordance with the Declaration of Helsinki (as revised in 2013). The study was approved by ethics board of Third Affiliated Hospital of Nantong university (NO. 2019029). Individual consent for this retrospective analysis was waived.

\section{Data collection}

The clinical records of enrolled patients were collected by 2 independent investigators. The collected data included age, sex, etiology of cirrhosis, etiology of bleeding, Child-Pugh score, model for end-stage liver disease (MELD) score, prior medication use, red blood cell (RBC) count, hemoglobin, white blood cell (WBC) count, platelets, albumin, aspartate transaminase (AST), alanine transaminase (ALT), total bilirubin, direct bilirubin, international normalized ratio (INR), antibiotic use, and units of packed RBCs required. In case of disagreement, a third investigator was invited to conduct a reassessment.

\section{Measurements of care}

In the traditional care group, patients were admitted to the intensive care unit for close observation and medication treatment after endoscopic procedures, until their condition was stable. Patients then received routine nursing care, such as symptomatic nursing, health education, and psychological counseling. After discharge, patients were followed up once a month for at least 6 months by telephone or internet to learn about their current condition and direct their self-care outside the hospital. 
In the MDTC group, a nursing-led multidisciplinary team was set up comprising of 1 digestive disease specialized nurse, 2 digestive disease attending physicians, 1 psychological consultant, a clinical dietitian, some normal nurses, and postgraduate volunteers. Generally, the digestive disease specialized nurse acted as the leader of the team to organize and coordinate the work of team members. The 2 attending physicians were responsible for assessing the condition of patients, analyzing the possibility of future development of UGIB, and providing suggestions for the next step of treatment and nursing care. The psychological consultant was responsible for assessing the current psychological status of patients and providing psychological therapy to improve their psychological status if necessary. The clinical dietitian was responsible for the analysis of the nutritional status of patients and providing suggestions for parenteral nutrition formulations. Nurses in this team were responsible for daily normal nursing care and telephone or online follow-up just as in the traditional care group, and postgraduate volunteers assisted in the collection and analysis of the clinical data. A short symposium was held once per week among the team to exchange experiences and problems, and several senior physicians were invited to evaluate and improve the work of the team.

\section{Outcomes}

The primary outcomes in this study were mortality and the incidence of rebleeding of enrolled patients within 1 year. Furthermore, quality of life was chosen as the secondary outcome. Quality of life was assessed according to the EuroQol 5-dimensions 5-levels questionnaire (EQ-5D-5L) within 4 weeks after the patients were discharged. The EQ-5D-5L was designed to assess 5 dimensions, including mobility, self-care, usual activities, pain and discomfort, and anxiety and depression of patients. The response of patients was divided into 5 levels: level 1 represented no problem and level 5 represented extreme problems. All responses were then converted into a score from 0 to 1,0 representing dead and 1 representing full health, according to a previous study (13).

\section{Statistical analysis}

Categorical values were represented as numbers and proportions, and compared using the chi-square test. Continuous variables were represented as mean and standard deviation, and were compared using the Student's $t$-test. Multivariable logistic regression was used to analyze the risk factors of mortality and rebleeding after adjusting for variables showing significant differences in the univariable logistic regression analysis. Similarly, multivariable linear regression was used to determine the effects of MDTC on the quality of life of enrolled patients. All analyses were performed using SPSS 22.0 software.

\section{Results}

As shown in Figure 1, 321 cirrhotic patients were admitted to our hospital during the research period. A total of 56 patients were excluded according to the exclusion criteria, resulting in 265 patients enrolled in this study. These patients were divided into the traditional care group and the MDTC group. During the follow-up period, 43 and 16 patients were lost to follow-up in the traditional care group and the MDTC group, respectively.

The clinical characteristics of the enrolled patients are shown in Table 1. The ages of patients in the traditional care group and the MDTC group were 57.5 \pm 13.6 and 55.1 \pm 13.9 , respectively, and both groups had more male patients than female patients. Hepatitis B virus (HBV) and alcohol were the 2 most common etiologies of cirrhosis in both groups. Compared with the traditional care group, patients in the MDTC group had significantly higher Child-Pugh scores and MELD scores (Child-Pugh score: 6.9 \pm 2.2 vs. $7.7 \pm 2.5, \mathrm{P}=0.02$; MELD score: $4.8 \pm 2.8$ vs. $5.8 \pm 3.6, \mathrm{P}=0.04$ ). Proton pump inhibitors (PPI) were the most used drugs in the enrolled patients. Some results of laboratory tests are also shown in Table 1. Patients in the MDTC group had a significantly lower level of WBCs and a significantly higher level of AST compared to the traditional care group (WBC: $7.0 \pm 2.4$ vs. $6.1 \pm 3.3, \mathrm{P}=0.01$; AST: $46.6 \pm 38.9$ vs. $58.7 \pm 41.0$, $\mathrm{P}=0.02)$. There were $9(8.9 \%)$ patients in the traditional care group and $15(14.3 \%)$ patients in the MDTC group receiving antibiotic prophylaxis, which showed no significant difference. Furthermore, patients received $2.8 \pm 1.0$ and $2.5 \pm 0.6$ units of packed RBCs in the traditional care group and the MDTC group, respectively.

The primary outcomes in this study were mortality and the incidence of rebleeding of enrolled patients within 1 year, as shown in Table 2. Patients in the MDTC group had a much lower incidence of rebleeding within 6 months and 1 year than the traditional care group $(\mathrm{P}=0.03$ and 0.02 , respectively). Also, the 1-year mortality in the MDTC group was significantly lower than in the traditional care group $(\mathrm{P}=0.01)$. Univariable and multivariable logistic 


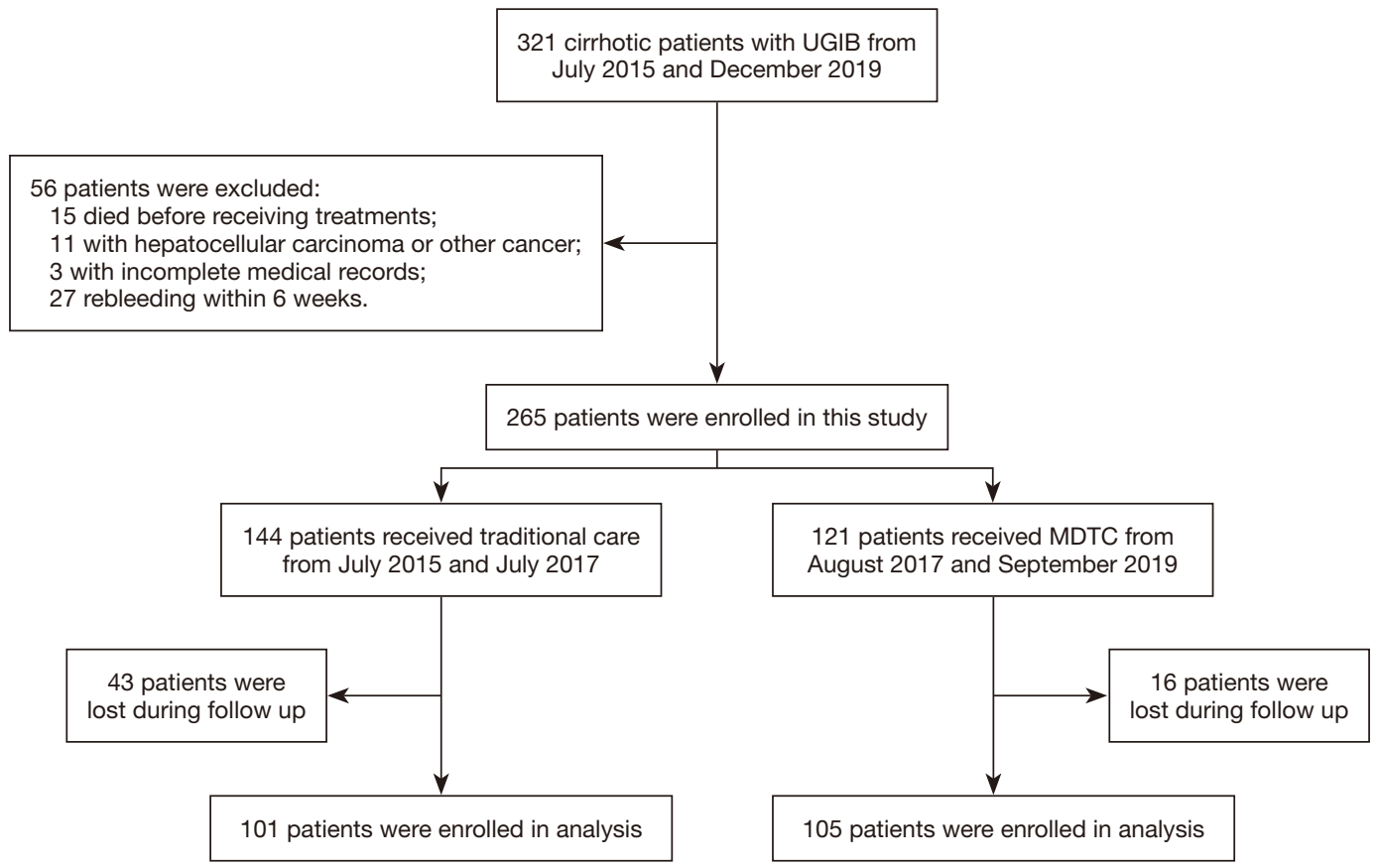

Figure 1 Flow chart of the study design. UGIB, upper gastrointestinal bleeding; MDTC, multidisciplinary team care.

regression were then performed to explore the risk factors of rebleeding and all-cause mortality within 1 year as shown in Table 3. MDTC, male sex, MELD score, units of packed RBCs required, albumin, hemoglobin, WBCs, and platelets were likely to be related to the incidence of rebleeding within 1 year according to the univariable logistic regression. After adjustment by multivariable logistic regression, MDTC was identified as a protective factor for rebleeding, and higher MELD score and more required units of packed RBCs were associated with a higher incidence of rebleeding. Similarly, MDTC was related to lower mortality of cirrhotic patients with UGIB according to the multivariable logistic regression analysis of risk factors of mortality. On the other hand, higher ChildPugh and MELD scores, more required units of packed RBCs, and lower platelet counts were associated with higher mortality.

The quality of life of enrolled cirrhotic patients with UGIB within 4 weeks after discharge was assessed using the EQ-5D-5L. The results shown in Table 4 indicated that patients in the MDTC group felt less discomfort and depression than those in the traditional care group (both $\mathrm{P}<0.01$ ). As for overall quality of life, patients in the MDTC group also had a significantly higher score than the traditional care group $(0.68 \pm 0.22$ vs. $0.76 \pm 0.24, \mathrm{P}=0.02)$.
The risk factors of quality of life in enrolled patients were then evaluated using multivariable linear regression, as shown in Table 5. MDTC was found to be related to improved quality of life, while older age and higher MELD scores were the risk factors of quality of life in cirrhotic patients with UGIB.

\section{Discussion}

Cirrhotic patients with UGIB usually have a high mortality rate and a poor prognosis. Therefore, how to improve the prognosis of patients warrants further investigation. The risk factors affecting mortality have been explored by several studies. Previous studies have demonstrated that age and sex were not associated with mortality in cirrhotic patients with UGIB $(6,8,14)$, consistent with the results of this study. Similarly, the etiologies of cirrhosis and bleeding were not related to the mortality of patients in our study, mainly because the majority of patients in this study had HBV- or alcohol-induced cirrhosis, and bleeding due to gastroesophageal varices. The most important risk factor for mortality in cirrhotic patients with UGIB was MELD score in this study, which represented the development of liver disease. Many studies have confirmed the MELD score as an important predictor of poor prognosis in UGIB patients 
Table 1 Characteristics of the enrolled cirrhotic patients with UGIB

\begin{tabular}{|c|c|c|c|}
\hline Variables & $\begin{array}{l}\text { Traditional } \\
\text { care group }\end{array}$ & $\begin{array}{l}\text { MDTC } \\
\text { group }\end{array}$ & $P$ value \\
\hline Number & 101 & 105 & \\
\hline Age, years & $57.5 \pm 13.6$ & $55.1 \pm 13.9$ & 0.83 \\
\hline Sex & & & 0.52 \\
\hline Male & $71(70.3 \%)$ & $78(74.3 \%)$ & \\
\hline Female & $30(29.7 \%)$ & $27(25.7 \%)$ & \\
\hline Etiology of cirrhosis & & & 0.61 \\
\hline HBV & $29(28.7 \%)$ & $32(30.5 \%)$ & \\
\hline HCV & $10(9.9 \%)$ & $4(3.8 \%)$ & \\
\hline Alcohol & $22(21.8 \%)$ & $29(27.6 \%)$ & \\
\hline $\mathrm{HBV}+\mathrm{HCV}$ & $5(5.0 \%)$ & $4(3.8 \%)$ & \\
\hline $\mathrm{HBV}+$ alcohol & $21(20.8 \%)$ & $16(15.2 \%)$ & \\
\hline $\mathrm{HCV}+$ alcohol & $4(4.0 \%)$ & $7(6.7 \%)$ & \\
\hline Drug-related & $2(2.0 \%)$ & $3(2.9 \%)$ & \\
\hline Autoimmune-related & $2(2.0 \%)$ & $1(1.0 \%)$ & \\
\hline Unknown & $6(5.9 \%)$ & $9(8.6 \%)$ & \\
\hline Etiology of bleeding & & & 0.56 \\
\hline Gastroesophageal varices & $91(90.1 \%)$ & 97 (92.4\%) & \\
\hline Peptic ulcer & $10(9.9 \%)$ & $8(7.6 \%)$ & \\
\hline Child-Pugh score & $6.9 \pm 2.2$ & $7.7 \pm 2.5$ & 0.02 \\
\hline MELD score & $4.8 \pm 2.8$ & $5.8 \pm 3.6$ & 0.04 \\
\hline Prior medication use & & & 0.90 \\
\hline PPI & $48(47.5 \%)$ & $56(53.3 \%)$ & \\
\hline NSAIDS & $20(19.8 \%)$ & $21(20.0 \%)$ & \\
\hline Statins & 15 (14.9\%) & $12(11.4 \%)$ & \\
\hline Antiplatelet agents & 13 (12.9\%) & $12(11.4 \%)$ & \\
\hline Anticoagulation & $5(5.0 \%)$ & $4(3.8 \%)$ & \\
\hline $\mathrm{RBC}, 10^{12} / \mathrm{L}$ & $3.2 \pm 1.0$ & $2.7 \pm 0.8$ & 0.33 \\
\hline Hemoglobin, g/L & $83.4 \pm 26.8$ & $81.3 \pm 26.5$ & 0.25 \\
\hline WBC, $10^{9} / \mathrm{L}$ & $7.0 \pm 2.4$ & $6.1 \pm 3.3$ & 0.01 \\
\hline Platelets, $10^{9} / \mathrm{L}$ & $114.1 \pm 76.5$ & $105 \pm 83.3$ & 0.12 \\
\hline Albumin & $30.7 \pm 8.9$ & $29.4 \pm 7.5$ & 0.29 \\
\hline AST & $46.6 \pm 38.9$ & $58.7 \pm 41.0$ & 0.02 \\
\hline ALT & $38.1 \pm 30.5$ & $41.2 \pm 35.5$ & 0.89 \\
\hline Total bilirubin & $36.4 \pm 41.2$ & $37.9 \pm 37.2$ & 0.30 \\
\hline
\end{tabular}

Table 1 (continued)
Table 1 (continued)

\begin{tabular}{lccc}
\hline Variables & $\begin{array}{r}\text { Traditional } \\
\text { care group }\end{array}$ & $\begin{array}{c}\text { MDTC } \\
\text { group }\end{array}$ & P value \\
\hline Direct bilirubin & $21.0 \pm 32.4$ & $24.5 \pm 39.7$ & 0.21 \\
INR & $1.4 \pm 0.3$ & $1.5 \pm 0.5$ & 0.78 \\
Antibiotic prophylaxis & $9(8.9 \%)$ & $15(14.3 \%)$ & 0.16 \\
$\begin{array}{l}\text { Units of packed RBC } \\
\text { required }\end{array}$ & $2.8 \pm 1.0$ & $2.5 \pm 0.6$ & 0.18 \\
\hline
\end{tabular}

HBV, hepatitis B virus; HCV, hepatitis C virus; MELD, model for end-stage liver disease; PPI, proton pump inhibitor; NSAIDS, nonsteroidal anti-inflammatory drugs; RBC, red blood cell; WBC, white blood cell; AST, aspartate transaminase; ALT, alanine transaminase; INR, international normalized ratio; MDTC, multidisciplinary team care; UGIB, upper gastrointestinal bleeding.

Table 2 Primary outcomes of the enrolled cirrhotic patients with UGIB

\begin{tabular}{lccc}
\hline Variables & $\begin{array}{c}\text { Traditional } \\
\text { care group }\end{array}$ & $\begin{array}{c}\text { MDTC } \\
\text { group }\end{array}$ & P value \\
\hline Rebleeding within 4 weeks & $5(5.0 \%)$ & $5(4.8 \%)$ & 0.95 \\
Rebleeding within 3 months & $15(14.9 \%)$ & $8(7.6 \%)$ & 0.10 \\
Rebleeding within 6 months & $27(26.7 \%)$ & $15(14.3 \%)$ & 0.03 \\
Rebleeding within 1 year & $38(37.6 \%)$ & $21(20.0 \%)$ & 0.02 \\
All-cause mortality in hospital & $8(7.9 \%)$ & $7(6.7 \%)$ & 0.73 \\
All-cause mortality within 1 year & $36(35.6 \%)$ & $20(19.0 \%)$ & 0.01 \\
\hline MDTC, multidisciplinary team care; UGIB, upper gastrointestinal \\
bleeding.
\end{tabular}

$(6,15,16)$. Peng et al. also revealed that Child-Pugh scores and MELD scores played similar roles in predicting the in-hospital mortality of cirrhotic patients with UGIB (17). Accordingly, Child-Pugh score was significantly associated with the mortality of enrolled patients in our study. The units of packed RBCs required were also reported to be related to the mortality of patients $(3,7)$. The results of our study also indicated that patients who received more units of packed RBCs had a higher mortality than those who received less.

The incidence of rebleeding was another important outcome in this study. Certain blood markers have been reported to be useful in predicting rebleeding in UGIB patients, such as C-reactive protein, prealbumin, and 
Table 3 Univariable and multivariable logistic regression of risk factors of rebleeding and all-cause mortality within 1 year

\begin{tabular}{|c|c|c|c|c|}
\hline Variables & \multicolumn{2}{|c|}{ Univariable } & \multicolumn{2}{|c|}{ Multivariable } \\
\hline \multicolumn{5}{|l|}{ Rebleeding } \\
\hline MDTC & $0.74(0.61-0.87)$ & 0.02 & $0.79(0.63-0.84)$ & 0.03 \\
\hline Sex, male/female & $1.46(1.16-1.94)$ & 0.03 & $1.51(0.87-2.62)$ & 0.14 \\
\hline Units of packed RBC required & $1.45(1.03-2.05)$ & 0.03 & $1.67(1.20-2.67)$ & 0.02 \\
\hline Albumin & $0.93(0.88-0.99)$ & 0.04 & $1.02(0.98-1.06)$ & 0.35 \\
\hline Hemoglobin & $0.92(0.88-0.96)$ & 0.01 & $0.93(0.85-1.02)$ & 0.12 \\
\hline WBC & $0.96(0.94-0.98)$ & 0.02 & $1.00(0.991 .01)$ & 0.38 \\
\hline MDTC & $0.63(0.45-0.91)$ & 0.01 & $0.79(0.59-0.98)$ & 0.04 \\
\hline Child-Pugh score & $1.34(1.16-1.52)$ & $<0.01$ & $1.22(1.12-1.32)$ & 0.02 \\
\hline MELD score & $2.01(1.20-3.37)$ & $<0.01$ & $2.34(1.31-3.59)$ & $<0.01$ \\
\hline Units of packed RBC required & $2.67(1.58-5.09)$ & 0.02 & $2.05(1.22-3.42)$ & 0.03 \\
\hline Albumin & $0.92(0.86-0.98)$ & 0.03 & $0.98(0.96-1.01)$ & 0.13 \\
\hline Hemoglobin & $0.99(0.98-0.99)$ & 0.04 & $1.00(0.99-1.01)$ & 0.61 \\
\hline Platelets & $0.93(0.89-0.97)$ & 0.02 & $0.95(0.92-0.99)$ & 0.04 \\
\hline
\end{tabular}

MDTC, multidisciplinary team care; OR, odds ratio; Cl, confidence interval; MELD, model for end-stage liver disease; RBC, red blood cell; WBC, white blood cell.

Table 4 Quality of life of the enrolled cirrhotic patients with UGIB within 4 weeks after discharge

\begin{tabular}{lccc}
\hline Variables & $\begin{array}{c}\text { Traditional } \\
\text { care group }\end{array}$ & $\begin{array}{c}\text { MDTC } \\
\text { group }\end{array}$ & P value \\
\hline Mobility & $2.1 \pm 0.6$ & $1.8 \pm 0.9$ & 0.08 \\
Self-care & $1.9 \pm 0.5$ & $1.9 \pm 0.6$ & 0.35 \\
Usual activities & $2.3 \pm 1.0$ & $2.1 \pm 0.7$ & 0.12 \\
Pain and discomfort & $2.9 \pm 1.0$ & $2.1 \pm 0.8$ & $<0.01$ \\
Anxiety and depression & $3.1 \pm 0.8$ & $1.9 \pm 1.1$ & $<0.01$ \\
Overall quality of life & $0.68 \pm 0.22$ & $0.76 \pm 0.24$ & 0.02 \\
\hline
\end{tabular}

MDTC, multidisciplinary team care; UGIB, upper gastrointestinal bleeding.

D-dimer $(18,19)$. However, we did not find a meaningful laboratory test indicator for rebleeding in this study. MELD score and required units of packed RBCs were the only 2 risk factors for rebleeding according to the multivariable logistic regression analysis.

Very few studies have explored the quality of life of UGIB patients. Campbell et al. conducted a prospective study in 2015 to investigate the situation of UGIB patients in the UK, and the results indicated that mean quality of life for survivors was 0.74 (20). However, the study did not further explore the risk factors for quality of life in these patients. In the present study, we were the first to demonstrate that older age and higher MELD score led to a poorer quality of life in cirrhotic patients with UGIB.

The most important aim of this study was to verify the protective role of MDTC in cirrhotic patients with UGIB. Our results indicated that MDTC could not only reduce mortality and the incidence of rebleeding, but also improve the quality of life of enrolled patients. Tsai et al. also found that MDTC could significantly reduce mortality in patients with breast cancer (21). The main reason why MDTC improved the prognosis of patients was that multidisciplinary cooperation and improved team 
Table 5 Univariable and multivariable linear regression of risk factors of quality of life in enrolled patients

\begin{tabular}{lcccc}
\hline \multirow{2}{*}{ Variables } & \multicolumn{2}{c}{ Univariable } & & \multicolumn{2}{c}{ Multivariable } \\
\cline { 2 - 3 } \cline { 5 - 6 } MDTC & Coefficient $(95 \% \mathrm{Cl})$ & P value & Coefficient $(95 \% \mathrm{Cl})$ & $-0.07(-0.12--0.02)$ \\
Age & $-0.06(-0.08--0.03)$ & 0.02 & $0.11(0.02-0.21)$ & 0.01 \\
MELD score & $0.08(0.04-0.12)$ & 0.01 & $0.06(0.02-0.12)$ & 0.03 \\
Units of RBCs required & $0.09(0.03-0.15)$ & $<0.01$ & $0.18(-0.06-0 f .42)$ & 0.26 \\
AST & $0.14(0.02-0.30)$ & 0.04 & $0.09(-0.10-0.28)$ & 0.34 \\
\hline
\end{tabular}

MDTC, multidisciplinary team care; MELD, model for end-stage liver disease; RBC, red blood cell; AST, aspartate transaminase; CI, confidence interval.

performance relieved the anxiety and depression of patients, as shown in Table 4. Improvements in the psychological status of patients increases compliance, which makes it easier for patients to cooperate with medical staff, leading to improvements in prognosis. Despite these advantages, there were still some problems with MDTC, including increased workload and lack of leadership. The digestive disease specialized nurse usually acted as the leader of the team to organize and coordinate the work of team members in this study. However, the increased workload made it hard for the specialized nurse to integrate teams and assign tasks in a timely manner. These problems have also been described in several previous studies, warranting further improvement in these processes $(22,23)$.

There were some limitations in this study. Firstly, this was a retrospective study. Some laboratory tests were not performed in the enrolled patients, such as prealbumin and D-dimer tests. This might have introduced bias into the multivariate analysis. Secondly, the EQ-5D-5L was used in this study to assess the quality of life of enrolled patients. EQ-5D-5L is one of the most commonly used scales to evaluate quality of life, however, it is relatively simple compared to some other scales, and can only provide limited information about patients. The use of scales that take less time but provide sufficient information would therefore be more beneficial for future studies. Thirdly, the follow-up period was one year, relatively short for the study, and we will prolong the follow-up period in the future.

\section{Conclusions}

In conclusion, this retrospective cohort study enrolled 206 cirrhotic patients with UGIB in order to analyze the effects of MDTC on prognosis and quality of life. As expected,
MDTC, compared with traditional care, could reduce the incidence of rebleeding and mortality not in the short term but over the long term. Furthermore, MDTC was useful for relieving anxiety and improving the quality of life of patients. Therefore, MDTC may be worth extending into several other fields.

\section{Acknowledgments}

Funding: This work was supported by Nantong Municipal Science and Technology Program (Guiding) Project (HS 149018) and Subject of Young Medical Personnel of Jiangsu Province (QNRC2016399).

\section{Footnote}

Reporting Checklist: The authors have completed the STROBE reporting checklist. Available at http://dx.doi. org/10.21037/apm-21-85

Data Sharing Statement: Available at http://dx.doi. org/10.21037/apm-21-85

Conflicts of Interest: All authors have completed the ICMJE uniform disclosure form (available at http://dx.doi. org/10.21037/apm-21-85). The authors have no conflicts of interest to declare.

Ethical Statement: The authors are accountable for all aspects of the work in ensuring that questions related to the accuracy or integrity of any part of the work are appropriately investigated and resolved. All procedures performed in this study involving human participants were in accordance with the Declaration of Helsinki (as revised 
in 2013). The study was approved by ethics board of Third Affiliated Hospital of Nantong university (NO. 2019029). Individual consent for this retrospective analysis was waived.

Open Access Statement: This is an Open Access article distributed in accordance with the Creative Commons Attribution-NonCommercial-NoDerivs 4.0 International License (CC BY-NC-ND 4.0), which permits the noncommercial replication and distribution of the article with the strict proviso that no changes or edits are made and the original work is properly cited (including links to both the formal publication through the relevant DOI and the license). See: https://creativecommons.org/licenses/by-nc-nd/4.0/.

\section{References}

1. Meng J, Xu H, Sui D, et al. A retrospective serological survey of hepatitis B virus infection in Northeast China. BMC Infect Dis 2019;19:440.

2. Wang H, Men P, Xiao Y, et al. Hepatitis B infection in the general population of China: a systematic review and meta-analysis. BMC Infect Dis 2019;19:811.

3. Fouad TR, Abdelsameea E, Abdel-Razek W, et al. Upper gastrointestinal bleeding in Egyptian patients with cirrhosis: Post-therapeutic outcome and prognostic indicators. J Gastroenterol Hepatol 2019;34:1604-10.

4. Lee EW, Shahrouki P, Alanis L, et al. Management Options for Gastric Variceal Hemorrhage. JAMA Surg 2019;154:540-8.

5. Xie W, Chen FX, Zhu LY, et al. Risk assessment of first upper gastrointestinal bleeding using computerized tomoscanning in esophageal varices patients with cirrhosis and portal hypertension. Medicine (Baltimore) 2020;99:e18923.

6. Li Y, Li H, Zhu Q, et al. Effect of acute upper gastrointestinal bleeding manifestations at admission on the in-hospital outcomes of liver cirrhosis: hematemesis versus melena without hematemesis. Eur J Gastroenterol Hepatol 2019;31:1334-41.

7. An Y, Bai Z, Xu X, et al. No Benefit of Hemostatic Drugs on Acute Upper Gastrointestinal Bleeding in Cirrhosis. Biomed Res Int 2020;2020:4097170.

8. Bishay K, Tandon P, Fisher S, et al. Clinical Factors Associated with Mortality in Cirrhotic Patients Presenting with Upper Gastrointestinal Bleeding. J Can Assoc Gastroenterol 2020;3:127-34.

9. Li P, Gong Y, Zeng G, et al. A new mode of community continuing care service for COPD patients in China: participation of respiratory nurse specialists. Int J Clin Exp Med 2015;8:15878-88.

10. Liang L, Pan Y, Wu D, et al. Effects of Multidisciplinary Team-Based Nurse-led Transitional Care on Clinical Outcomes and Quality of Life in Patients With Ankylosing Spondylitis. Asian Nurs Res (Korean Soc Nurs Sci) 2019;13:107-14.

11. Yesil S, Akinci B, Bayraktar F, et al. Reduction of major amputations after starting a multidisciplinary diabetic foot care team: single centre experience from Turkey. Exp Clin Endocrinol Diabetes 2009;117:345-9.

12. Sharifzadeh Y, Slade AN, Weiss E, et al. Attitudes and Perceptions Towards Radiation Therapy in Breast Cancer Patients: The Role of a Multidisciplinary Care Team. J Cancer Educ 2020. [Epub ahead of print]. doi: 10.1007/ s13187-019-01677-1.

13. Luo N, Liu G, Li M, et al. Estimating an EQ-5D-5L Value Set for China. Value Health 2017;20:662-9.

14. Xavier SA, Vilas-Boas R, Boal Carvalho P, et al. Assessment of prognostic performance of Albumin-Bilirubin, ChildPugh, and Model for End-stage Liver Disease scores in patients with liver cirrhosis complicated with acute upper gastrointestinal bleeding. Eur J Gastroenterol Hepatol 2018;30:652-8.

15. Zou D, Qi X, Zhu C, et al. Albumin-bilirubin score for predicting the in-hospital mortality of acute upper gastrointestinal bleeding in liver cirrhosis: A retrospective study. Turk J Gastroenterol 2016;27:180-6.

16. Hsu SC, Chen CY, Weng YM, et al. Comparison of 3 scoring systems to predict mortality from unstable upper gastrointestinal bleeding in cirrhotic patients. Am J Emerg Med 2014;32:417-20.

17. Peng Y, Qi X, Dai J, et al. Child-Pugh versus MELD score for predicting the in-hospital mortality of acute upper gastrointestinal bleeding in liver cirrhosis. Int J Clin Exp Med 2015;8:751-7.

18. Yue $W$, Liu $Y$, Jiang $W$, et al. Prealbumin and D-dimer as Prognostic Indicators for Rebleeding in Patients with Nonvariceal Upper Gastrointestinal Bleeding. Dig Dis Sci 2020. [Epub ahead of print]. doi: 10.1007/s10620-02006420-1.

19. Lee HH, Park JM, Lee SW, et al. C-reactive protein as a prognostic indicator for rebleeding in patients with nonvariceal upper gastrointestinal bleeding. Dig Liver Dis 2015;47:378-83.

20. Campbell HE, Stokes EA, Bargo D, et al. Costs and quality of life associated with acute upper gastrointestinal bleeding in the UK: cohort analysis of patients in a cluster 
randomised trial. BMJ Open 2015;5:e007230.

21. Tsai CH, Hsieh HF, Lai TW, et al. Effect of multidisciplinary team care on the risk of recurrence in breast cancer patients: A national matched cohort study. Breast 2020;53:68-76.

22. Luo Y, Huang Y, Chen X, et al. Effects of Multidisciplinary Team Care Based on 5E's Renal Rehabilitation for Peritoneal Dialysis Patients in Guangxi Zhuang

Cite this article as: Wang S, Qian X. Effect of multidisciplinary team care on the management of cirrhotic patients with upper gastrointestinal bleeding: a retrospective cohort study. Ann Palliat Med 2021;10(3):3050-3058. doi: 10.21037/apm-21-85
Autonomous Region of China: A Randomized Controlled Trial. Blood Purif 2019;48:115-23.

23. Zhong XM, Wen XH, Ji CH, et al. A temporarysustainable team: A new multidisciplinary team model for severe trauma. Chin J Traumatol 2020;23:363-6.

(English Language Editor: C. Betlazar-Maseh) 